\title{
Performance Comparison of Artificial Intelligence Techniques for Non-intrusive Electrical Load Monitoring
}

\author{
Khairuddin Khalid $^{1}$, Azah Mohamed ${ }^{2}$, Ramizi Mohamed ${ }^{3}$, Hussain Shareef ${ }^{4}$ \\ ${ }^{1,2,3}$ Centre for Integrated Systems Engineering and Advanced Technologies, Faculty of Engineering and Built \\ Environment, Universiti Kebangsaan Malaysia, Bangi, 43600, Malaysia \\ ${ }^{4}$ College of Engineering, United Arab Emirates University, P O Box 15551, Al Ain, United Arab Emirates
}

\begin{tabular}{l} 
Article Info \\
\hline Article history: \\
Received March 13, 2018 \\
Revised May 14, 2018 \\
Accepted May 28, 2018 \\
\hline
\end{tabular}

\section{Keywords:}

Artificial Neural Network (ANN)

Extreme learning machine Non-intrusive load monitoring Support vector machine

\begin{abstract}
The increased awareness in reducing energy consumption and encouraging response from the use of smart meters have triggered the idea of nonintrusive load monitoring (NILM). The purpose of NILM is to obtain useful information about the usage of electrical appliances usually measured at the main entrance of electricity to obtain aggregate power signal by using a smart meter. The load operating states based on the on/off loads can be detected by analysing the aggregate power signals. This paper presents a comparative study for evaluating the performance of artificial intelligence techniques in classifying the type and operating states of three load types that are usually available in commercial buildings, such as fluorescent light, airconditioner and personal computer. In this NILM study, experiments were carried out to collect information of the load usage pattern by using a commercial smart meter. From the power parameters captured by the smart meter, effective signal analysis has been done using the time time (TT)transform to achieve accurate load disaggregation. Load feature selection is also considered by using three power parameters which are real power, reactive power and the TT-transform parameters. These three parameters are used as inputs for training the artificial intelligence techniques in classifying the type and operating states of the loads. The load classification results showed that the proposed extreme learning machine (ELM) technique has successfully achieved high accuracy and fast learning compared with artificial neural network and support vector machine. Based on validation results, ELM achieved the highest load classification with $100 \%$ accuracy for data sampled at 1 minute time interval.
\end{abstract}

Copyright $(2018$ Institute of Advanced Engineering and Science. All rights reserved.

\section{Corresponding Author:}

Ramizi Mohamed,

Centre for Integrated Systems Engineering and Advanced Technologies

Faculty of Engineering and Built Environment

Universiti Kebangsaan Malaysia, Bangi, 43600, Malaysia

e-mail: azah_mohamed@ukm.edu.my

\section{INTRODUCTION}

Monitoring energy consumption of electrical appliances in various building is an important part of energy management with advantages such as knowing energy consumption behavior, and for troubleshooting. By implementing efficient energy management, user demand management by the utility may lead to considerable energy savings. However, conventional load monitoring has several disadvantages due to the use of expensive meter and high installation cost, thus making it unsuitable for large scale monitoring. Therefore, non-intrusive load monitoring (NILM) based on monitoring electrical loads at one point of power measurement is introduced. This method is economical because it does not require installation of many meters and has potential for better energy management. 
Currently, smart meter with NILM has been used by power supply companies to evaluate uses of electric power in consumer homes. NILM is defined as the separation process of individual electrical equipment in an electrical circuit by analyzing the signal obtained at a point of measurement of power [1]. In general, the idea of implementing NILM is to separate the aggregate energy obtained from smart meters to the energy of individual electrical equipment [2]. The implementation process of the NILM method is simple because it does not require the use of large number of meters and that only one smart meter is installed at the main entrance of electricity. However, it is necessary to pass through a complex process of signal separation analysis to separate the aggregate signals monitored by smart meters to signals of individual equipment used [3]. By implementing the NILM method, consumers are able to monitor the operation of all electrical loads used in a building without incurring high installation cost of meters.

In NILM, it is also important to predict and classify the operation of various electrical appliances [4]. For predicting the operation of various electrical appliances, different artificial intelligence and machine learning techniques have been used such as artificial neural network (ANN), fuzzy logic and support vector machine (SVM) [5]. By using artificial intelligence techniques, the operation of the associated electrical appliances can be classified. Some researchers have implemented NILM using the ANN classification technique [6] while SVM technique was used in [7], [8]. The random forests method is also considered in the prediction of electricl loads to identify the type and status of loads [9]. In addition, some researchers applied the nearest neighbors in predicting loads in NILM [10]. However, the load prediction accuracy is still unsatisfactory and therefore there is a need to develop better and accurate prediction technique.

In this paper, a performance comparison is made on the use of artificial intelligence techniques to monitor three types of electrical appliances with different load profile conditions. The three electrical appliances considered are the load types that are usually available in commercial buildings, such as fluorescent light, air-conditioner and personal computer. The compared artificial intelligence techniques are the extreme learning machine (ELM), ANN and SVM techniques.

\section{THE MATERIAL AND METHOD}

The entire NILM implementation procedure can be divided into two parts as shown in Figure 1. The first part is data collection and analysis of load profiles while the second part is the selection and extraction of load features and the development of artificial intelligence techniques to predict and classify the loads.

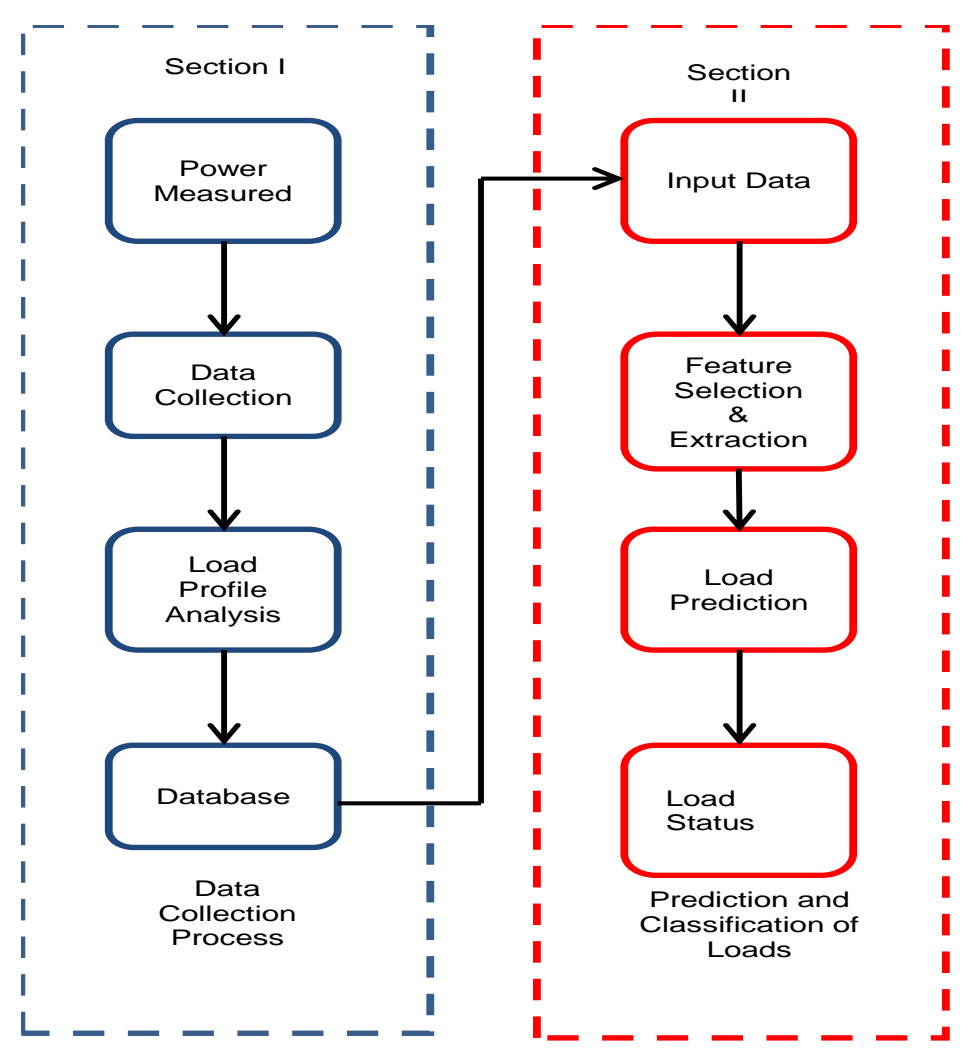

Figure 1 Procedures in the proposed NILM 


\subsection{Load Profile Data Collection and Analysis}

NILM is implemented in a real laboratory scenario by installing a smart meter at the distribution panel which is the main entrance of power source. Figure 2 shows the proposed NILM system developed at the laboratory scale. The electrical appliances that were monitored are the fluorescent lights, air conditioners and personal computers which create different patterns of power consumption. The smart meter installed in the system monitor 7 sets of fluorescent lights, one air conditioner unit and several personal computers. These appliances are switched ON and OFF within 24 hours. Load monitoring is performed by using the supervised method in which all targeted individual load signals are recorded along with the aggregate signals. However, for 24 hours of electrical equipment such as the computer server, its power is ignored and the operation of this computer is not considered in this NILM study

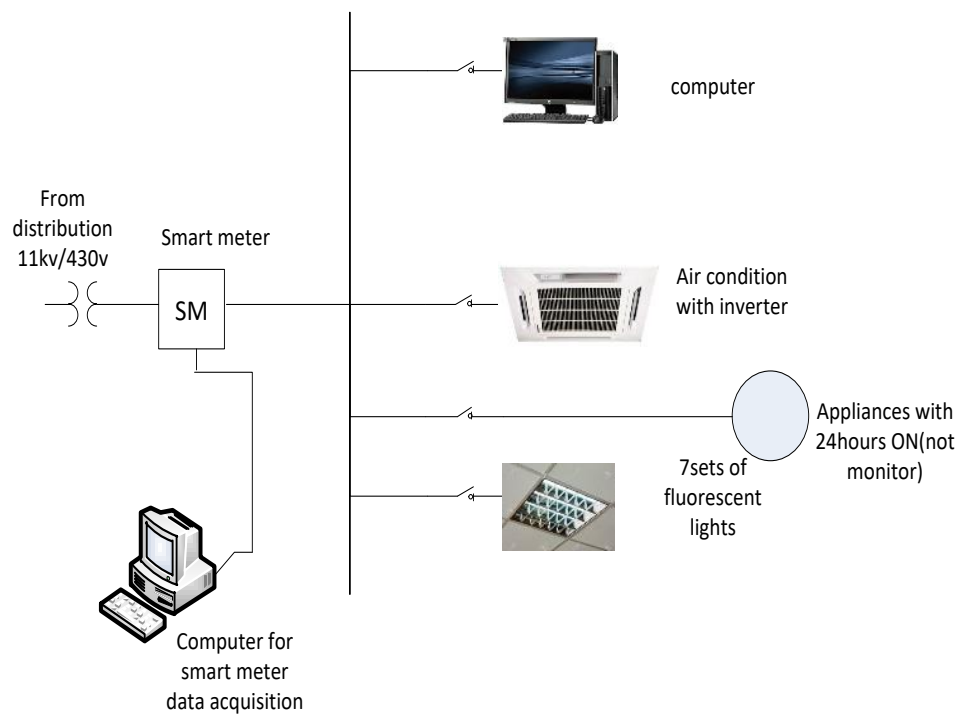

Figure 2. Set-up of the proposed NILM system

In the implementation of NILM, it is important to carry out load profile analysis to ensure that all the monitored loads can be identified. The load profile measured is the overall shape of the operation of the device in terms of power $(\mathrm{P})$ against time $(\mathrm{t})$. The data collected should include the overall pattern shown by the nature of the load. This is to ensure the accuracy of the load model trained with artificial intelligence techniques.

Figure 3 shows the real power measured by the smart meter at sampling rates of 1, 10 and 30 minutes. These sampling rates are considered to assess the capability of the smart meter in disaggregating loads at low and high sampling rates. Considering the energy usage profile and taking into account the maximum demand at a certain period of time, the maximum number of appliances in use is identified.

The power parameters recorded by the smart meter are grouped in the form of tables according to the data sampling by using the EnerSis software. The complete data collected for each record is within 24 hours based on maximum demand within a certain interval. All data obtained from the smart meter, such as active power, reactive power, power factor, voltage and current are collected in a database for further analysis and classification of load types. Data collection process is done by considering all combinations of open and close operation of the three types of electrical equipment. 


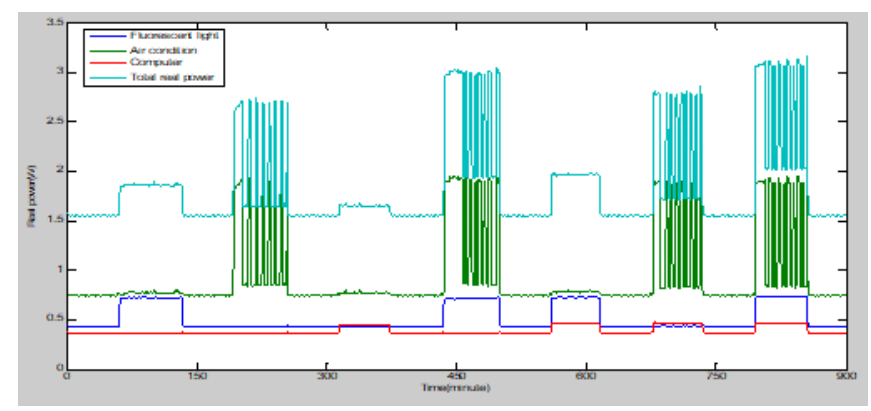

(a)

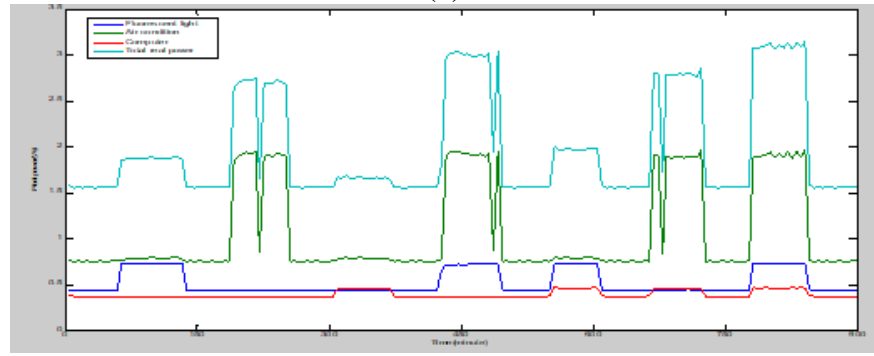

(b)

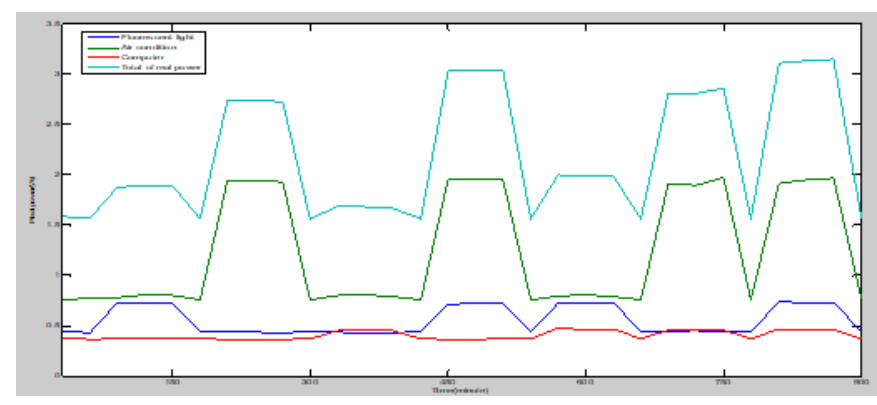

(c)

Figure 3. Real power measured at (a) 1, (b) 10 and (c) 30 minute sampling rates

Data collected into databases are divided into two sets of data randomly selected, ie, $60 \%$ as training data for training while $40 \%$ of samples for testing the artificial intelligence techniques. From the experiments performed, the data sets obtained are such that 1706 samples for 1 minute data, 170 samples for 10 minute data and 56 samples for 30 minute data. The collected data were obtained by means of supervised load monitoring while the validation data were collected by taking into account the opening and closing of electrical equipment at different times. The number of data sets collected for training, testing and validating the artificial intelligence techniques are 549, 366 and 1706 samples respectively.

\subsection{Load Feature Selection and Extraction}

Selection and extraction of the features of the three types of electrical equipment are performed to select the best load features for the purpose of identifying the opening and closing of load operations. By using the commercial smart meters, we can obtain power parameters such as real power (W), reactive power (Var) and power factor. All of these parameters are measured automatically by smart meters and selected as the load features. To improve the load recognition accuracy, an additional feature is extracted from the smart meter data by using a relatively advanced signal processing technique known as the time-time (TT)-transform [11].The TT-transform is based on the S-transform [12] and it is a time-time analysis technique which is able to localize the signal. It is used for feature extraction by creating a different feature of the load profile for non-periodic electrical signals. The TT-transform technique applied on a non-periodic signal is considered appropriate because the appliance switching event is easily detected with respect to time. The TT-transform signal is a 2 dimensional time-time representation of a 1 dimensional time signal and it is given by [13], 


$$
T_{T}(t, \tau)=\int_{-\infty}^{\infty} S(\tau, f) \exp (2 i \pi f t) d f
$$

where $\mathrm{f}$ is the frequency, $\mathrm{t}$ and $\tau$ are the time variables.

The TT-transform analysis is used to detect events occuring in the signal so as to provide information about the appliance activity. The event caused by switching activity is localized by the time features of the real power around a particular point on the time axis. The new time feature provides a better event detection occuring during the time period at a certain energy level and magnitude. Both the TTtransform and $\mathrm{S}$-transform produce $\mathrm{m} \times \mathrm{n}$ matrices, in which the $\mathrm{S}$-transform is in the time-frequency domain whereas the TT-transform is in the time domain. Both the S-transform and TT-transform are complex numbers and can be used as features to classify the non-periodic signals obtained from a smart meter. The information in the TT-transform matrix can be plotted as time-time mesh and contours so as to facilitate the analysis in signal changing detection via visual inspection of the energy level. From the complex numbers of each element in the TT-transform, several parameters are derived using the standard statistical analysis. The T-transform features are analysed by calculating statistical indices such as maximum, minimum, standard deviation, and mean. The standard deviation is selected because it is a robust feature that can enhance the accuracy of load identification. The standard deviation is computed and derived from the T-transform matrix as it gives a good feature to classify the target appliance. Thus, the load features selected and extracted in this work are the real and reactive powers and the TT-transform standard deviation. The correlation between the event occurring in the signal and the T-transform analysis is shown in Figures 4 and 5.

Figure 4 shows example of the absolute values of the TT-transformation of real active power signal according to different load operations. From this figure, the different types of loads and power levels show different patterns of contour. This comparison can be seen through the resulting pattern and the resulting color level in which the pattern reflects the current events as well as the Open and Close operation of the loads.

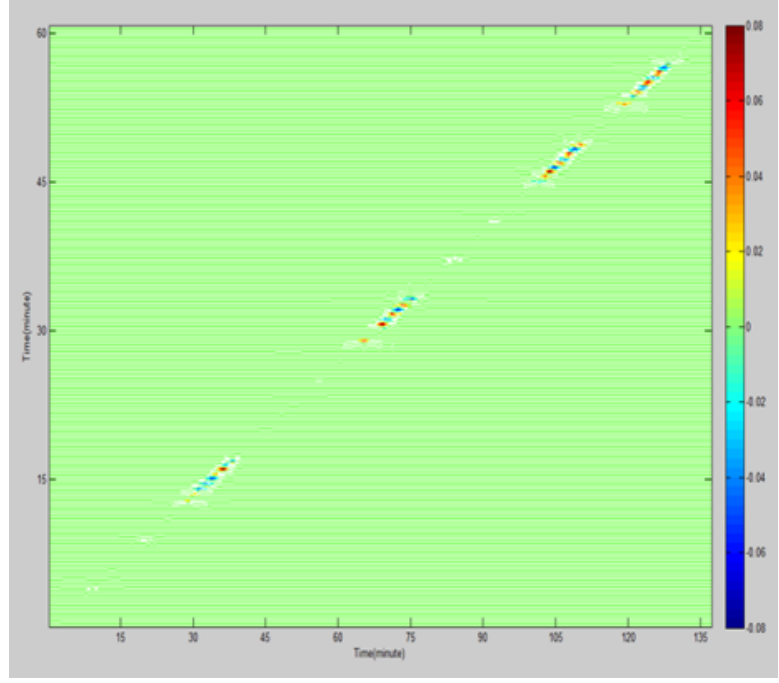

Figure 4. TT-transformed of an example load power signal

To detect the load operation events more precisely, the overall picture in the form of mesh plot is given as in Figure 5. With a 3-dimensional plot, the difference in power usage for each load combination can be seen more clearly where each load combination gives different dimensions of power generation. 


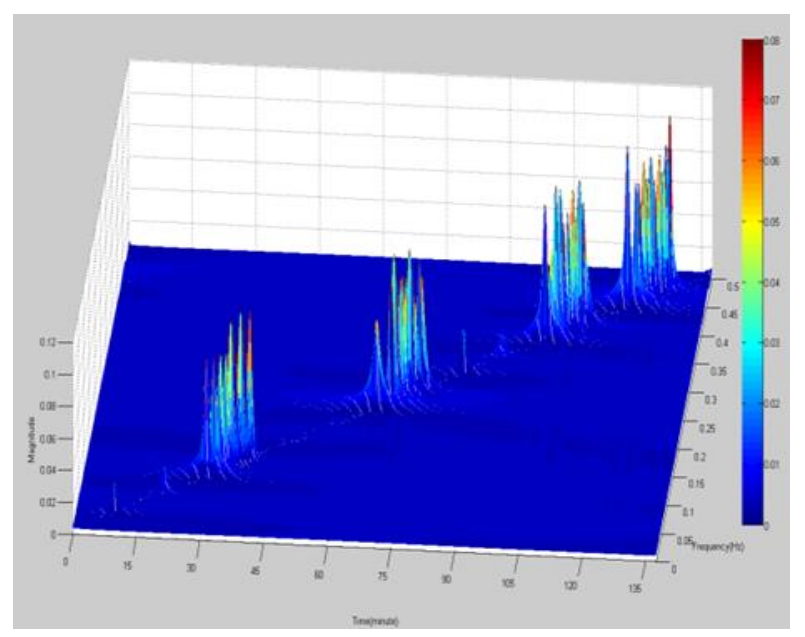

Figure 5. The 3 dimensional plot for detecting the load switching events

\subsection{Classification of Loads Using Artificial Intelligence Techniques}

In this study, three artificial intelligence techniques are considered to identify the type and status of load operations for NILM application. The artificial intelligence techniques considered are the extreme learning machine (ELM), artificial neural network (ANN) and support vector machine (SVM).

ELM is based on the generalized single layer feedforward neural network in which the hidden layer does not require tuning [14]. It is developed to address the slow learning speed problems of the gradient based learning algorithm. The number of hidden nodes in ELM is less compared to the number of input nodes. The single layer feedforward neural network with randomly chosen input weights and hidden layer biases can exactly learn $\mathrm{N}$ hidden nodes with distinct observation [15]. The ELM reproduces the input patterns at the output layer through minimizing the reconstruction error. The algorithm starts with selecting the parameter randomly in the hidden nodes and the output weights are analytically determined through a simple generalized inverse operation of the hidden layer output matrices. The output layer weights are obtained by using the least square method. In ELM, it is unnecessary to perform iterative tuning in which the parameters of the hidden nodes, input weights and biases are generated randomly and the output weights are analytically computed by means of a simple generalized inverse operation. The ELM method randomly generates input and hidden layers connected weights and hidden layer neuron threshold. Table 1 shows the results of the optimum parameters for the ELM technique. The selection of hidden neurons is performed by systematic iteration method using the sin activation function. of sin.

Table 1. Optimum Parameters of ELM

\begin{tabular}{cc}
\hline Parameter & Optimum Parameter \\
\hline Number of hidden neurons & 33 neurons $-1 \mathrm{~min}$ \\
& 22 neurons $-10 \mathrm{~min}$ \\
& 13 neurons $-30 \mathrm{~min}$ \\
Activation function & $\sin$ \\
\hline
\end{tabular}

ANN was also implemented for the development of the NILM model to predict the type of load and its operation. The RNT model considers the multi-layer feedforward model. The NILM implementation procedure using the multi-layer ANN starts by setting the data set which consists of input and output data for training. The optimum ANN model is achieved by determining the optimum values for hidden neurons and learning rates based on the trial and error approach. The mean square errors are evaluated and the optimum parameters for the multi-layer ANN are determined as shown in Table 2. For a sampling rate of 1 minute, 4 neurons and learning rate of 0.5 are the optimum parameters with $100 \%$ ANN accuracy. Furthermore, the optimum values of hidden neurons and optimum learning rates for sampling rate of 10 minutes and 30 minutes are 4 neurons and 0.1 and 6 neurons and 0.9 , respectively. 
Table 2. ANN Parameters

\begin{tabular}{cc}
\hline Parameter & Parameter Value \\
\hline Mean square error (MSE) & 0.0001 \\
Optimum no of hidden neurons & 4 neurons $-1 \mathrm{~min}$ \\
& 3 neurons $-10 \mathrm{~min}$ \\
Optimum learning rate & 6 neurons $-30 \mathrm{~min}$ \\
& $0.1-1 \mathrm{~min}$ \\
Hidden layer & $0.5-10 \mathrm{~min}$ \\
Hidden neuron activation function & $0.9-30 \mathrm{~min}$ \\
Output neuron activation function & 1 \\
\hline
\end{tabular}

Support vector machine (SVM) is one of the techniques used for the load classification in NILM [7]. Three SVM models have developed to predict three types of targeted loads. To form a robust SVM model, optimization of two parameters, namely, gamma, $\gamma$ and sigma, have been considered. The gamma parameter, $\gamma$ is the parameter of the compiler that determines the suitability between error and smoothness while sigma refers to the open space of the basic function of the kernel radius. An iterative optimization technique has been implemented to determine both of these optimum parameters by considering $\gamma$ values set between 0.1 and 10 and sigma values in the range 0.01 to 1 . Table 3 shows the optimum parameters of the SVM kernel function. For a sampling rate of 1 minute, $\gamma=1.1$ and sigma $=0.11$ were selected as the optimum values for training the SVM. Furthermore, for a sampling rate of 10 minutes, the optimum values for sigma=0.11 and $\gamma=1.1$ while for a sampling rate of 30 minutes optimum values of $\gamma=0.1$ and sigma $=0.11$.

Table 3. Optimum Parameters for SVM

\begin{tabular}{lll}
\hline Parameter & Value & Sampling rate \\
\hline Gamma, $\gamma$ & 1.1 & $1 \mathrm{~min}$ \\
& 1.1 & $10 \mathrm{~min}$ \\
& 0.1 & $30 \mathrm{~min}$ \\
Sigma, $\sigma^{2}$ & & \\
& 0.11 & $1 \mathrm{~min}$ \\
& 0.11 & $10 \mathrm{~min}$ \\
Kernel function type & 0.11 & $30 \mathrm{~min}$ \\
\hline
\end{tabular}

To evaluate the performance of the developed ELM, ANN and SVM developed, errors for target and predicted outputs need to be assessed. The mean squared error (MSE) has been used as a performance index to evaluate the binary output errors in which the binary output is in terms of the load appliance operation which is labeled as ' 1 ' or ' 0 ' referring to the ON and OFF states, respectively. The MSE equation is given as follows:

$$
\mathrm{MSE}=\frac{1}{n} \sum_{i=1}^{n}\left(Y_{i}-\hat{Y}_{i}\right)^{2} .
$$

where is the predicted output for each data set $\mathrm{n}$, and $\mathrm{Y}$ is the target output.

The performance of the developed model is also assessed based on the correct classification and misclassification of the training and testing data. Evaluation of the result is based on the load output which is classified as true positive (TP), and false negative (FN) are expressed as follows:

$\mathrm{TP}=\Sigma \mathrm{t}($ Actual output $\&$ Predicted output $=\mathrm{ON}$

$\mathrm{FN}=\Sigma \mathrm{t}($ Actual output $=\mathrm{ON} \&$ Predicted output $=\mathrm{OFF})$

From the correct classification and misclassification of the training and testing data, the accuracy is determined as follows:

Accuracy $=(\mathrm{TP}+\mathrm{FN}) /($ No of overall outputs $)$

In addition, the F-score is considered in which it refers to the average accuracy and also recovery (Hyungsul Kim et al. 2011). F-score is expressed as, 
where precision is the number of correct positive results divided by the number of all positive results returned by the classifier, and recall is the number of correct positive results divided by the number of all relevant samples (all samples that should have been identified as positive).

\section{RESULTS AND ANALYSIS}

Input data for training the ELM, ANN and SVM considers the active power (P), reactive power (Q) and the resulting TT-transform standard deviation obtained from the load features. The ELM, ANN and SVM output is the operating status of the type of lights, air conditioner and personal computer labeled as ' 1 ' for open load operation and ' 0 ' for load-closing operation. Input and output data are generated and collected from load switching activities by considering three data sampling rates, ie, 1 minute, 10 minutes and 30 minutes. Table 4 shows the number of data sets used for training, testing and validating the ELM, ANN and SVM.

Table 4. Data sets for ELM, ANN and SVM

\begin{tabular}{cccc}
\hline \multirow{2}{*}{ Type of Data } & \multicolumn{3}{c}{ Sampling Time } \\
\cline { 2 - 4 } & $1 \mathrm{~min}$ & $10 \mathrm{~min}$ & $30 \mathrm{~min}$ \\
\hline Training & 549 & 55 & 18 \\
Testing & 366 & 36 & 12 \\
Validation & 1706 & 170 & 56 \\
Total & 2621 & 261 & 86 \\
\hline
\end{tabular}

All the ELM, ANN and SVM have been trained using the optimum parameters. The training and testing results for the ELM, ANN and SVM are presented in the following sub-sections.

\subsection{ELM, ANN and SVM Training Results}

The ELM, ANN and SVM were trained using data sets with sampling rates of 1, 10 and 30 minutes and the training results are as shown in Table 5. Performance evaluation of the trained ELM, ANN and SVM is based on the MSE, percentage overall accuracy and F-score calculations. Based on the training results obtained, the ELM, ANN and SVM give good predictive results with almost $100 \%$ overall accuracy. By comparing the training results, ELM gives the best prediction with MSE=0, F-score=1, and 100\% overall accuracy when compared to the ANN and SVM techniques.

Table 5. ELM, ANN and SVM Training Results

\begin{tabular}{lllll}
\hline Sampling & Parameter & \multicolumn{3}{l}{ Prediction Accuracies } \\
\cline { 3 - 5 } Rate & & ELM & ANN & SVM \\
\hline 1 min & MSE & 0 & 0.0012 & 0.0027 \\
& $\begin{array}{l}\text { Overall } \\
\text { Accuracy (\%) }\end{array}$ & 100 & 99.88 & 99.73 \\
& F-score & 1 & 0.996 & 0.995 \\
\hline $10 \mathrm{~min}$ & MSE & 0 & 0.0121 & 0 \\
& Overall & 100 & 98.79 & 100 \\
& Accuracy (\%) & & & \\
& F-score & 1 & 0.98 & 1 \\
\hline 30 min & MSE & 0 & 0 & 0 \\
& Overall & 100 & 100 & 100 \\
& Accuracy (\%) & & & \\
& F-score & 1 & 1 & 1 \\
\hline
\end{tabular}

The best overall accuracy is given by ELM with $100 \%$ accuracy for the three sampling rates, the next is SVM with $100 \%$ accuracy for the 10 minutes and 30 minutes sampling rates and then followed by ANN with $100 \%$ accuracy for the 30 minutes sampling rate. However, there are slight inaccuracies in prediction for SVM and ANN as shown in Table 5. False prediction occurs may be due to the undefined pattern of signals at certain sampling rates and there is a confusion in determining the output by the developed technique. 


\subsection{ELM, ANN and SVM Testing Results}

To evaluate the effectiveness of ELM, ANN and SVM in predicting and classifying, testing and validation were performed using $40 \%$ testing data sets and other new data sets for validation purposes. The testing data sets were selected randomly to avoid using the same training data set so as to test the effectiveness of the developed artificial intelligence techniques. Furthermore, the data sets used for validation is a new data set that reflects the pattern of signals grouped according to the usage of loads. As such, this validation can evaluate the actual effectiveness of the developed models. Table 6 shows the overall results of the testing and validation performed on the ELM, ANN, and SVM.

Table 6 ELM, ANN and SVM Testing and Validation Results

\begin{tabular}{cccccccc}
\hline $\begin{array}{c}\text { Sampling } \\
\begin{array}{c}\text { Rate } \\
(\mathrm{min})\end{array}\end{array}$ & Technique & \multicolumn{2}{c}{ MSE } & \multicolumn{2}{c}{$\begin{array}{c}\text { Overall } \\
\text { Accuracy } \%\end{array}$} & F-Score \\
\hline \multicolumn{2}{c}{1} & Test & Val & Test & Val & Test & Val \\
\hline & ELM & 0 & 0 & 100 & 100 & 1 & 1 \\
& ANN & 0.001 & 0.004 & 99.82 & 99.77 & 0.994 & 0.997 \\
& SVM & 0.001 & 0 & 99.94 & 99.98 & 0.999 & 0.999 \\
\hline 10 & ELM & 0 & 0.018 & 100 & 98.24 & 1 & 0.983 \\
& ANN & 0.028 & 0.157 & 98.15 & 95.69 & 0.957 & 0.948 \\
& SVM & 0.009 & 0.022 & 99.07 & 97.84 & 0.982 & 0.978 \\
\hline 30 & ELM & 0 & 0.012 & 100 & 97.62 & 1 & 0.948 \\
& ANN & 0.028 & 0.427 & 97.22 & 93.45 & 0.970 & 0.948 \\
& SVM & 0.028 & 0.054 & 97.22 & 94.64 & 0.970 & 0.958 \\
\hline
\end{tabular}

Val-Validation

Based on the results obtained, initial observations indicate that accuracy decreases with increase in the sampling rates from 1 minute to 30 minutes. This decrease in accuracy occurs due to loss of information about the unique features of the load contained in the aggregate power signal. In addition, the power signal at 30 minutes sampling time does not show significant changes between the combined power of the aggregate load and this can cause confusion to predict the load classification. The performance of the ELM technique using the testing data set indicates an overall accuracy of $100 \%$ for all the sampling rates. However, there is a slight decrease in accuracy in the validation results in which the overall accuracy are $98.24 \%$ and $97.62 \%$ for sampling times of 10 minutes and 30 minutes, respectively.

From the testing results shown in Table 6, SVM is seen to be the second best technique by giving overall accuracies of $99.94 \%, 99.07 \%$ and $97.22 \%$ for sampling times of 1,10 and 30 minutes, respectively. However, the validation results for SVM showed a slight decrease in accuracy with $97.84 \%$ and $94.64 \%$ for sampling rates of 10 minutes and 30 minutes, respectively. The third best is the ANN technique where the testing overall accuracies are $99.82 \%, 98.15 \%$ and $97.22 \%$ according to the three sampling rates. There was a decrease in accuracy in the ANN validation results, ie, $99.77 \%, 96.69 \%$ and $93.45 \%$ for sampling rates of 1 , 10 and 30 minutes, respectively. In general, the ELM technique gives the best results in terms of overall accuracy in load classification compared to the SVM and ANN.

\section{CONCLUSION}

This paper has presented a comparative study for evaluating the performance of artificial intelligence techniques in classifying the type and operating states of three load types, namely, fluorescent light, air-conditioner and personal computer for NILM. In the development of the NILM, a smart meter is used to measure real power at sampling rates of 1,10 and 30 minutes and artificial intelligence techniques such as ELM, ANN and SVM are used to predict and classify the loads that are in operation. To enhance the load identification accuracy, additional input features to the ELM, ANN and SVM have been used, namely, the TT-transform features. The ELM, ANN and SVM were trained and tested using measured data from the smart meter at various sampling rates. Evaluation of the effectiveness of ELM, ANN and SVM techniques is measured in terms of MSE, overall percentage accuracy, and F-score values. Comparing the results of the ELM, ANN and SVM in predicting the operation of the three load types, the ELM technique has given more accurate and efficient results than other techniques in terms of accuracy of output predictions. Results have proven the effectiveness of the ELM in load classification by obtaining overall percentage accuracy of $100 \%, \mathrm{MSE}=0$ and $\mathrm{F}$-score of 1 during testing and validation for sampling rate of $1 \mathrm{~min}$. 


\section{ACKNOWLEDGEMENT}

We would like to thank Universiti Kebangsaan Malaysia for providing the financial support on this project under Project Code GP-K009665.

\section{REFERENCES}

[1] G. W. Hart, "Nonintrusive appliance load monitoring," IEEE Proceedings, 80(12), pp.1870-1891, 1992.

[2] D. Egarter and W. Elmenreich, "Autonomous load disaggregation approach based on active power measurements," in First IEEE Workshop on Pervasive Energy Services PerEnergy, pp. 1-8, 2015.

[3] H. Ahmadi, and J. R. Marti, "Load decomposition at smart meters level using eigenloads approach," IEEE Transactions on Power Systems, 30(6), pp. 3425-3436, 2015.

[4] K. Basu, V. Debusschere, S. Bacha, U. Maulik and S. Bondyopadhyay, "Nonintrusive load monitoring: A temporal multilabel classification approach," IEEE Transactions on Industrial Informatics, 11(1), pp. 262-270, 2015.

[5] R. Bonfigli, S. Squartini, M. Fagiani and F. Piazza, "Unsupervised algorithms for non-intrusive load monitoring: An up-to-date overview”, in Proc IEEE 15th International Conference on Environment and Electrical Engineering, EEEIC 2015, pp. 1175-1180, 2015.

[6] D. Srinivasan, W. S. Ng, and A. C. Liew, "Neural-network-based signature recognition for harmonic source identification," IEEE Transactions on Power Delivery, 21(1), 398-405, 2006.

[7] L. Jiang, J. Li, S. Luo, S.West and G. Platt, "Power load event detection and classification based on edge symbol analysis and support vector machine," Applied Computational Intelligence and Soft Computing, 1-10, 2012.

[8] L. Du, Y. Yang, D. He, R. G. Harley, T. G. Habetler and B. Lu, "Support vector machine based methods for nonintrusive identification of miscellaneous electric loads," in Proc. IECON 2012, 2012, pp. 4866-4871.

[9] J. Mei, D. He, R. G. Harley and T. G. Habetler, "Random forest based adaptive non-intrusive load identification", in Proc. International Joint Conference on Neural Networks, 2014, pp. 1978-1983.

[10] M. B. Figueiredo, A. De Almeida, B. Ribeiro and A. Martins, "Extracting features from an electrical signal of a non-intrusive load monitoring system," Lecture Notes in Computer Science (including subseries Lecture Notes in Artificial Intelligence and Lecture Notes in Bioinformatics), Vol. 6283, pp. 210-217, 2010.

[11] C. Simon, M. Schimmel and J. J. Dañobeitia, "On the TT-transform and its diagonal elements," IEEE Transactions on Signal Processing, 56(11), pp. 5709-5713, 2008.

[12] C. R. Pinnegar and L. Mansinha, "The S-transform with windows of arbitrary and varying shape," Geophysics, 68(1), pp. 381, 2003.

[13] G. B. Huang, Q. Zhu, C. Siew and G. H., Zhu, "Extreme learning machine: Theory and applications," Neurocomputing, 70(1-3), 489-501, 2006.

[14] E. Cambria, and Guang-Bin, "Extreme Learning Machines", IEEE Intelligent Systems, 28(6), pp. 30-59, 2013.

[15] O. Kramer, O. Wilken, P. Beenken, A. Hein, A. Hüwel, T. Klingenberg and C. Meinecke, "On ensemble classifiers for nonintrusive appliance load monitoring," Lecture Notes in Computer Science (including subseries Lecture Notes in Artificial Intelligence and Lecture Notes in Bioinformatics), Vol. 7208, pp. 322-331, 2012. 